\title{
Application of ANSYS Finite Element Analysis in Teaching of Mechanics of Materials
}

\author{
Wang Feng ${ }^{1,2}$ MA Yurong ${ }^{1, *}$ Jiang Yaqiong $^{1}$ Li Dan $^{1}$ Liao Haifei ${ }^{1}$ \\ ${ }^{1}$ College of Civil and Environmental Engineering, Anhui Xinhua University, Hefei 230088, China \\ ${ }^{2}$ Key Laboratory of Building Structure of Anhui Higher Education Institutes, Anhui Xinhua University, Hefei 230088, China
}

\begin{abstract}
The traditional classroom teaching of mechanics of materials focuses on the theoretical derivation of the formula, and the content is relatively boring. However, in the experimental class, the calculation formula of bending normal stress is simply verified, and other information other than deformation can not be given directly. In order to improve the effect of classroom teaching, ANSYS finite element analysis and pure bending experiment are combined and applied to the classroom teaching of material mechanics. The deformation and stress in the deformation problem of material mechanics are vividly displayed in the form of animation and cloud chart. Practice has proved that this teaching method can not only deepen students' understanding of abstract mechanical concepts, but also stimulate students' interest in learning and innovative thinking, which is conducive to the cultivation of engineering application-oriented talents.
\end{abstract}

\section{Introduction}

Mechanics of materials is a very important professional basic course in application-oriented universities. It is closely combined with engineering practice and plays an important role in improving students' ability to solve engineering problems and innovation ability. At present, the traditional teaching content of mechanics of materials tends to focus on theoretical analysis, lack of advanced teaching means of modern technology, relatively boring content, and students' perceptual knowledge is insufficient. Therefore, how to effectively improve the teaching effect of mechanics of materials has been one of the hot spots in the teaching reform of mechanics [2]. At present, numerical simulation has made initial development in the teaching of mechanics of materials [3-5], but it still needs further research.

Bending deformation is one of the basic deformations of components in mechanics of materials, which often occurs in beam members. The normal stress produced by bending deformation is the key factor affecting the strength and stiffness of beam members. Therefore, the derivation of the formula of normal stress of beam section is often regarded as the important and difficult content of teaching. In the textbook of mechanics of materials, three aspects of deformation geometry, physical relations and statics are comprehensively considered to derive the formula of bending normal stress. The whole derivation process is very logical and involves many mathematical formulas. Students often find it difficult to understand abstract mechanical concepts and profound mechanical formulas [6]. In addition, in the process of beam bending normal stress electrical measurement experiment, students can not observe the deformation and stress distribution of the beam in the elastic range with naked eyes, so there are some difficulties in understanding the bending deformation process, and the lack of perceptual knowledge leads to students' lack of interest in the rational understanding of bending normal stress.

In order to improve students' interest in learning this part of the content, at the same time enhance students' ability to analyze and solve problems and innovative thinking, enrich the teaching content, this paper combined with the beam bending normal stress electrical measurement experiment, and added the finite element analysis results, using ansys 14.5 software to draw the stress and strain nephograms of beam bending deformation, and combine the finite element calculation with experimental analysis and theoretical calculation The results show that the theoretical formula is correct and the teaching effect is significantly improved.

\section{Electrical measurement of normal bending stress of beams}

In the case of pure bending of the beam, based on the assumption that there is no extrusion between the longitudinal fibers and the plane assumption, the calculation formula of bending normal stress is obtained as follows

$$
\sigma=\frac{M y}{I_{Z}}
$$

\footnotetext{
* Corresponding author: 2518946794@qq.com
} 
Where, $M$ is the bending moment, $I_{Z}$ is the moment of inertia of the transverse plane of the beam and $y$ is the ordinate of the stress location point.

It can be seen from formula (1) that when the beam is in pure bending state, the normal stress at each point changes linearly along the cross-section height. Assuming that there is no extrusion between the longitudinal fibers, the element in the pure bending beam is in the state of simple tension or compression. According to Hooke's law $\sigma=E \varepsilon$ of unidirectional stress state, as long as $\varepsilon$ of different beam heights are measured, the stress $\sigma$ of this point can be calculated.

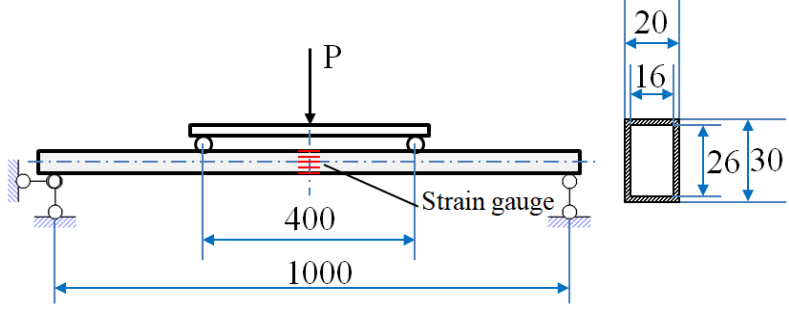

Fig. 1 Pure bending experiment of beam

The schematic diagram of pure bending test of the beam is shown in Fig. 1. The cross section of the beam is hollow rectangular section, the material is low carbon steel, and the elastic modulus is $\mathrm{E}=70 \mathrm{GPa}$. In the experiment, the incremental loading method is used. The load sensor directly measures the pressure exerted on the beam, and the bending moment of the pure bending section of the beam is obtained by calculation. On the side and upper and lower surfaces of the mid span position of the beam, five resistance strain gauges are pasted along different beam heights parallel to the axis, and the strain gauges are equidistant. The neutral axis position on the beam is taken as 0 , and the strain gauges are respectively located at the heights of $15 \mathrm{~mm}, 5 \mathrm{~mm}, 0$, $-5 \mathrm{~mm}$ and $-15 \mathrm{~mm}$ (corresponding to $\mathrm{A} 1 \sim \mathrm{A} 5$ in Table 1 ). The strain at this position is measured by the resistance strain gauge, and the stress at the height of the beam can be obtained. The experimental data and processing results are shown in Table 1 . The error between the experimental value and the theoretical value of stress at each point is less than $5 \%$, which verifies the correctness of the theoretical formula. In the whole experimental process, students strictly follow the steps, but only get the strain and stress values of several points, so they can not directly observe the deformation and stress distribution of the beam, and they still lack perceptual understanding of the deformation process.

\section{ANSYS finite element analysis}

In order to overcome the above difficulties in material mechanics theory and experimental teaching, and make students easy to understand and master more abstract mechanical knowledge, the finite element analysis software is used to simulate the experimental calculation examples, and the abstract concepts and conclusions are expressed in the form of graphics, so as to visualize the extraction knowledge and promote the students' mastery of mechanical knowledge.

Table 1 Experimental data and processing results

\begin{tabular}{|c|c|c|c|c|c|c|c|c|c|c|c|}
\hline \multirow{2}{*}{\multicolumn{2}{|c|}{ Load $(\mathbf{k N})$}} & \multicolumn{10}{|c|}{ Resistance strain gauge reading $(\mu \varepsilon)$} \\
\hline & & \multicolumn{2}{|c|}{ A1 } & \multicolumn{2}{|c|}{ A2 } & \multicolumn{2}{|r|}{ A3 } & \multicolumn{2}{|c|}{ A4 } & \multicolumn{2}{|c|}{ A5 } \\
\hline$P$ & $\triangle \mathrm{P}$ & $\varepsilon$ & $\Delta \varepsilon$ & $\varepsilon$ & $\Delta \varepsilon$ & $\varepsilon$ & $\Delta \varepsilon$ & $\varepsilon$ & $\Delta \varepsilon$ & $\varepsilon$ & $\Delta \varepsilon$ \\
\hline 0 & \multirow{2}{*}{0.050} & 0.0 & \multirow{2}{*}{-70.0} & 0.0 & \multirow{2}{*}{-32.0} & 0.0 & \multirow{2}{*}{2.4} & 0.0 & \multirow{2}{*}{15.9} & 0.0 & \multirow{2}{*}{63.7} \\
\hline 0.050 & & -70.0 & & -32.0 & & 2.4 & & 15.9 & & 63.7 & \\
\hline & 0.050 & & -72.1 & & -28.2 & & -1.6 & & 21.8 & & 70.1 \\
\hline 0.100 & & -142.1 & & -60.2 & & 0.8 & & 37.7 & & 133.8 & \\
\hline & 0.050 & & -75.5 & & -20.3 & & -9.5 & & 31.8 & & 74.4 \\
\hline & 0.050 & & -71.6 & & -23.0 & & \multirow{2}{*}{-8.7} & & 32.5 & & 80.3 \\
\hline 0.200 & & -289.2 & & -103.5 & \multirow[b]{2}{*}{-23.1} & -13.9 & & 102.0 & & 288.5 & \\
\hline 0.250 & 0.050 & -372.1 & -82.9 & $\mid-126.6$ & & -17.0 & -3.1 & 129.7 & 27.7 & 365.6 & 77.1 \\
\hline \multicolumn{2}{|c|}{$\begin{array}{c}\text { Experimental value } \\
\text { of } \Delta \varepsilon \\
\end{array}$} & -74 & & -25 & & & -4.10 & 25 & .94 & 73 & 12 \\
\hline $\begin{array}{r}\text { Experim } \\
\text { of } \sigma\end{array}$ & $\begin{array}{l}\text { tal value } \\
\text { (pa) }\end{array}$ & -5.2 & & -1.7 & & & -0.287 & 1.8 & 816 & 5.1 & 118 \\
\hline $\begin{array}{r}\text { Theoret } \\
\text { of } \sigma\end{array}$ & $\begin{array}{l}\text { l value } \\
\text { (pa) }\end{array}$ & -5.2 & & -1.7 & & & 0 & 1.7 & 739 & 5.2 & 217 \\
\hline Err & (\%) & -0 . & & 1.9 & & & / & 4. & 43 & -1 & .90 \\
\hline
\end{tabular}

Using ANSYS software, according to the size of experimental specimen in Fig. 1, beam 189 element type is selected to establish the model of hollow rectangular section beam, as shown in Fig. 2. The elastic modulus $\mathrm{E}=70 \mathrm{GPa}$, Poisson's ratio $\mu=0.3$. According to the experimental conditions of pure bending beam, the constraint and load are applied, and then the statics solution is carried out, and the calculation results can be displayed intuitively through post-processing.

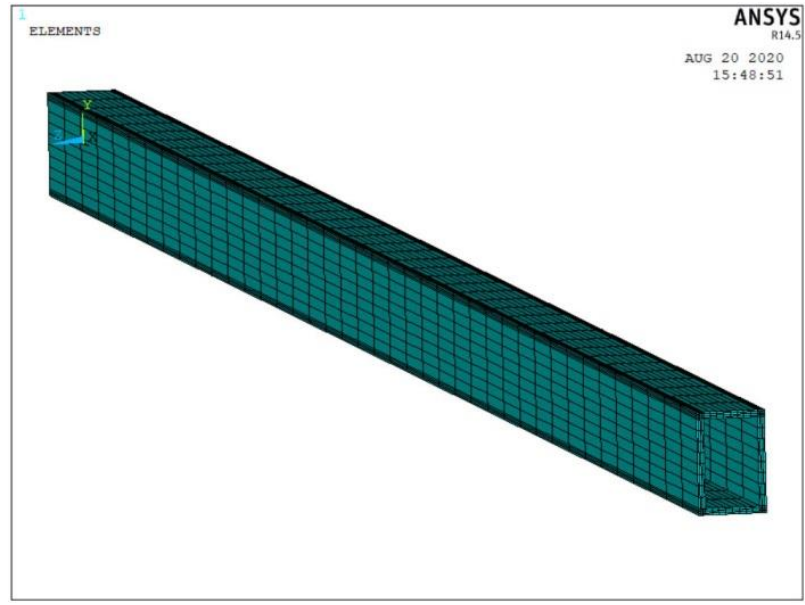

Fig. 2 Beam model

Fig. 3 shows the bending moment diagram of the beam, which is consistent with the theoretical calculation result. The bending moment of the beam segment between the two loads is a constant value of $7.5 \mathrm{~N} \cdot \mathrm{m}$, that is, the deformation of the beam segment is pure bending. Fig. 4 shows the deflection curve of beam axis 
calculated by ANSYS. It is easy to see that the maximum deflection at the mid span position is $0.549 \mathrm{~mm}$, which is difficult to observe through the experiment.

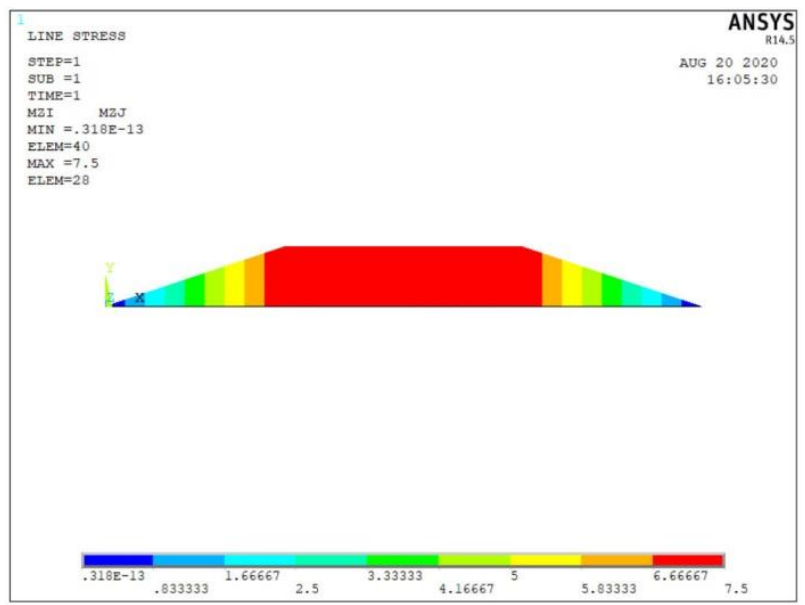

Fig. 3 Beam bending moment diagram

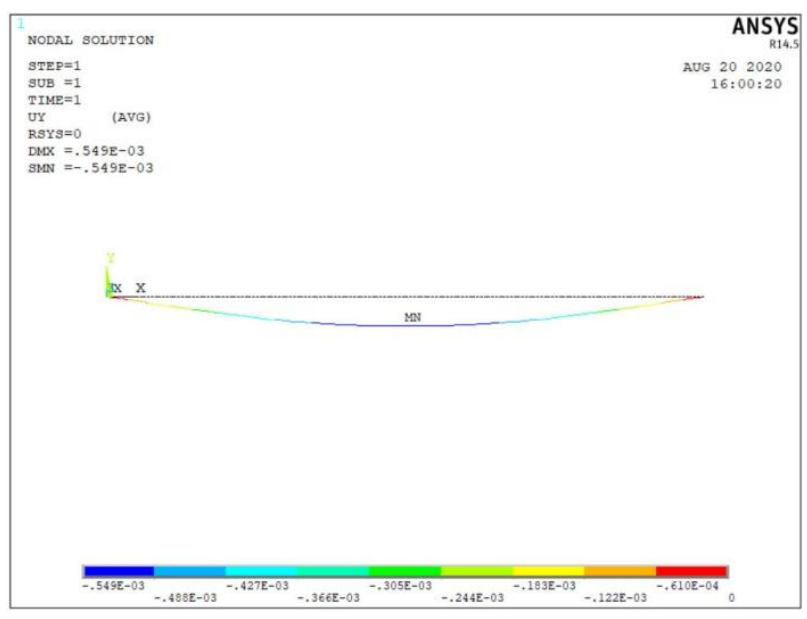

Fig. 4 Deflection curve of beam axis

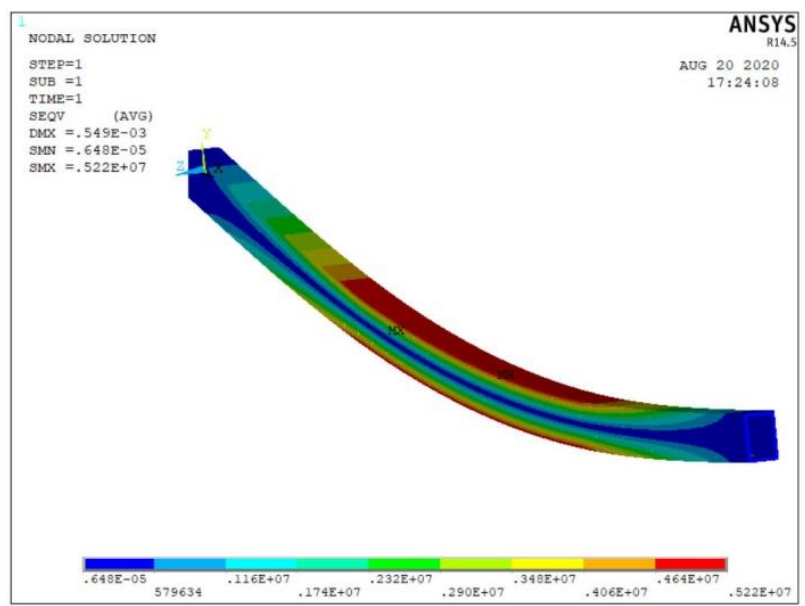

Fig. 5 Equivalent stress nephogram of beam

Fig. 5 shows the equivalent stress nephogram of the beam. It can be seen from the figure that the maximum value of the equivalent stress is located at the upper and lower edges of the middle constant bending moment beam section, and the equivalent stress gradually decreases when it approaches the two ends, which indicates that the normal stress is proportional to the bending moment.

Fig. 6 shows the equivalent strain nephogram of the beam. Similar to Fig. 5, the maximum equivalent strain occurs at the upper and lower edges of the middle beam segment, and the maximum value is $0.745 \times 10^{-4}$.

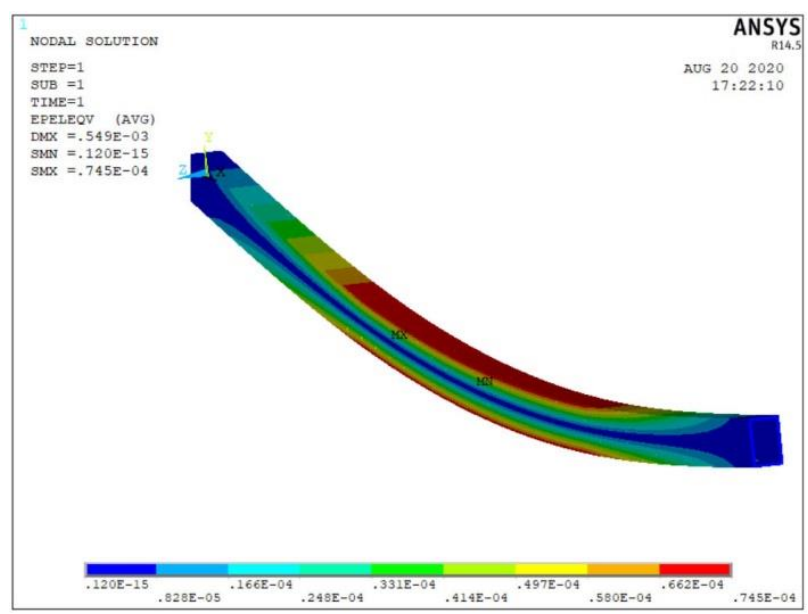

Fig. 6 Equivalent strain nephogram of beam

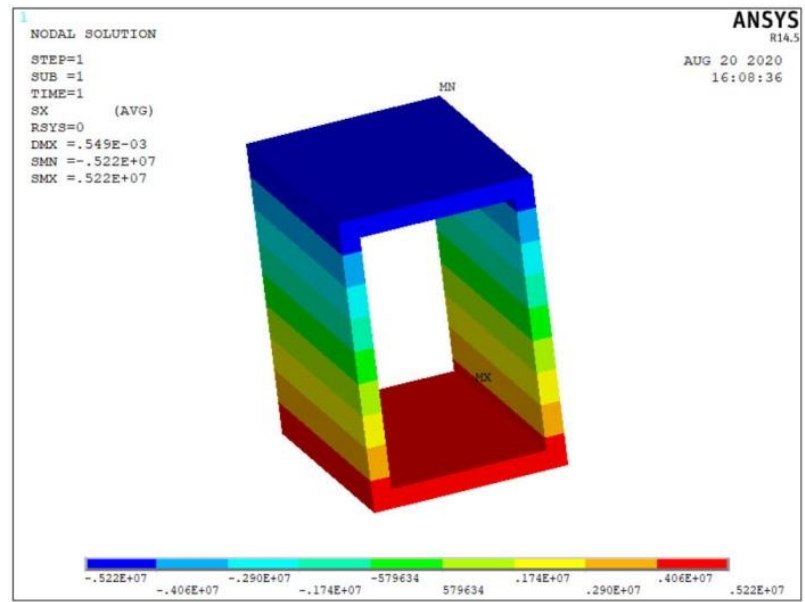

Fig. 7 Stress nephogram of beam mid span element

Fig. 7 shows the stress nephogram of the mid span element of the beam. It can be seen that the farther the beam is from the neutral layer, the greater the stress value, with the maximum value of $5.220 \mathrm{MPa}$, which is in good agreement with the maximum stress of $5.217 \mathrm{MPa}$ obtained by formula (1). The maximum tensile stress is $5.220 \mathrm{MPa}$, located at the lower edge. The maximum compressive stress is $-5.220 \mathrm{MPa}$, located at the upper edge. At the neutral layer, the stress is close to 0 . The stress distribution chromaticity along the height of the beam presents the rule of layered change. It is also proved that the normal stress at any point in the cross section is directly proportional to the distance from the point to the neutral axis when the beam is subjected to pure bending deformation, that is, the normal stress at each point on the same transverse line with the same distance from the neutral axis on the cross section is equal. In addition, it can be seen from the stress diagram that the cross-section of the pure bending beam does not warp after bending deformation, and it still remains 
plane, which also shows that the pure bending deformation of the beam conforms to the plane assumption.

Through the experiment and ANSYS analysis, the stress values of 5 points on the pure bending beam are obtained, and compared with the theoretical value, see Table 2. It can be seen from the data in the table that the error between the stress value obtained by the two different methods and the theoretical value is smaller, while the calculated value of ANSYS is closer to the theoretical value, which shows that the experimental analysis, finite element calculation and theoretical formula are unified, which fully proves the correctness of the theoretical formula.

Table 2 Comparison of three calculation results of pure bending beam section stress

\begin{tabular}{|c|c|c|c|c|c|}
\hline Measuring position & $\mathrm{A} 1$ & $\mathrm{~A} 2$ & $\mathrm{~A} 3$ & $\mathrm{~A} 4$ & $\mathrm{~A} 5$ \\
\hline $\begin{array}{c}\text { Experimental value } \\
\text { of } \boldsymbol{\sigma} \text { (Mpa) }\end{array}$ & -5.209 & -1.772 & -0.287 & 1.816 & 5.118 \\
\hline $\begin{array}{c}\text { ANSYS calculation } \\
\text { value of } \boldsymbol{\sigma}(\mathrm{Mpa})\end{array}$ & -5.220 & -1.740 & 0.000 & 1.740 & 5.220 \\
\hline $\begin{array}{c}\text { Theoretical value of } \\
\boldsymbol{\sigma}(\mathrm{Mpa})\end{array}$ & -5.217 & -1.739 & 0 & 1.739 & 5.217 \\
\hline
\end{tabular}

\section{Conclusion}

In this paper, the finite element technology is introduced into the teaching of mechanics of materials to enhance the visibility of the analysis results. Students can see the distribution law of stress diagram and strain diagram intuitively. In addition, the change process of stress and strain can also be made into animation for teaching demonstration, which increases the interest of teaching. In this way, students' interest in learning is improved, the difficulty of learning theoretical formula is reduced, and the ability of analyzing and solving problems is enhanced. Through the comparison of experimental analysis, ANSYS analysis and theoretical calculation results, the three are unified, which fully proves the correctness and practicability of the theoretical calculation formula.

\section{Acknowledgements}

This work was financially supported by Key Project of Natural Science Research in Universities of Anhui Province (KJ2019A0882), Anhui Province Quality Engineering Project (2019jxtd118) and Universities Quality Engineering Project (2019jy031).

\section{References}

1. Zhang Hongwei, Xi Jun, $\mathrm{Xu}$ Yuemei, et al. Discussion on Application of Finite Element Numerical Simulation in Teaching for Materials Mechanics. Higher Education in Chemical Engineering, 2016, 33(02):80-84(in Chinese)
2. Zhou Xiaomin, Sun Zheng. ANSYS and teaching of mechanics of materials. Mechanics in Engineering, 2019, 41(2):222-226(in Chinese)

3. Qin Keli. Application of finite element analysis software in material mechanics simulation experiment. Journal of irrigation and drainage, 2007, 26(S1):60-61(in Chinese)

4. KAO Si-ming, LU Xiao-yu, GONG Neng-ping. On Application of Numerical Simulation in Teaching of Material Mechanics. Journal of Southwest China Normal University(Natural Science Edition), 2014, 39(12):209-211(in Chinese)

5. Long Lianchun, Liu Haihong, Yang Qingsheng. Numerical simulation analysis system for basic concepts of material mechanics. Experimental Technology and Management, 2013, 30(08):8689(in Chinese)

6. XU Bing, YIN Guansheng, SHE Bin, et al. Teaching Method Research on Metarial Mechanics by FEA Method. Experiment Science and Technology, 2018, 16(01):82-85(in Chinese) 